\title{
HPV e câncer de cabeça e pescoço: desenvolvimento de um aplicativo para adolescentes
}

\section{HPV and head and neck cancer: development of an application for adolescents}

\author{
Alcir Humberto Rodrigues \\ Universidade Estadual do Centro-Oeste (UNICENTRO)
}

Elaine Fátima Brek

Universidade Estadual do Centro-Oeste (UNICENTRO)

Flavia Valenga

Universidade Estadual do Centro-Oeste (UNICENTRO)

Cleverson Sebastião dos Anjos

Instituto Federal de Educação, Ciência e Tecnologia do Paraná (IFPR)

Valter Luís Estevam Junior

Instituto Federal de Educação, Ciência e Tecnologia do Paraná (IFPR)

David Livingstone Alves Figueiredo

Universidade Estadual do Centro-Oeste (UNICENTRO)

Cristina Ide Fujinaga

Universidade Estadual do Centro-Oeste (UNICENTRO)

\begin{abstract}
Resumo: A adolescência é uma fase marcada por grandes transformações, que envolvem aspectos biológicos, psíquicos, sociais e culturais. Nesse sentido, o adolescente apresenta vulnerabilidades para a transmissão das doenças sexualmente transmissíveis, entre elas o HPV. Constata-se o aumento da prevalência do câncer de cabeça e pescoço em adultos jovens, associada ao HPV. Assim, o objetivo da pesquisa foi o desenvolvimento de um aplicativo para smartphones, voltado à adolescentes, como estratégia de prevenção do câncer de cabeça e pescoço causado pelo HPV. Para tal, realizou-se oficinas participativas com 90 adolescentes de ensino médio, com idade entre 16 e 18 anos, sobre a relação entre câncer de cabeça e pescoço e HPV. O conteúdo do aplicativo foi elaborado segundo temas levantados pelos alunos. $\mathrm{O}$ conteúdo do aplicativo foi validado por expertises, com concordância maior que $85 \%$. A validação de aparência foi realizada pelos próprios adolescentes, com o mesmo nível de concordância.
\end{abstract}

Palavras-chave: Aplicativos Móveis. Câncer. Educação Sexual. HPV. Prevenção.

Abstract: Adolescence is a phase characterized by great changes, that involves biological, psychic, social and cultural aspects. In this context, adolescents show vulnerabilities for the transmission of sexually transmitted diseases, including the HPV. It is observed an increase in 
the prevalence of head and neck cancer in young adults associated with HPV. Therefore, the objective of the research was the development of mobile phone application software, aimed at adolescents, as a strategy to prevent head and neck cancer caused by HPV. To that end, participatory workshops were performed with 90 high school adolescents, aged 16-18 years, about the relationship between head and neck cancer and HPV. The content of the mobile phone application software was elaborated according to topics approached by students. Mobile phone application software content was validated by experts, with agreement greater than $85 \%$. The validation of appearance was performed by adolescents themselves, with the same level of agreement.

Keywords: Mobile Applications. Cancer. Sexual Education. HPV. Prevention.

RODRIGUES, Alcir Humberto; BREK, Elaine Fátima; VALENGA, Flavia; ANJOS, Cleverson Sebastião dos; JUNIOR, Valter Luís Estevam; FIGUEIREDO, David Livingstone Alves; FUJINAGA, Cristina Ide. HPV e câncer de cabeça e pescoço: desenvolvimento de um aplicativo para adolescentes. Informática na Educação: teoria \& prática, Porto Alegre, v. 22, n. 2, p. 58-73, maio./ago. 2019. 


\section{Introdução}

A adolescência é uma fase marcada por grandes transformações, que envolvem aspectos biológicos, psíquicos, sociais e culturais. Nesse período, ocorre ainda a iniciação sexual, que possibilita ao adolescente fazer escolhas (in)conscientes, considerando desejos, prazeres e riscos (MORAES, VITALLE, 2012). De acordo com a UNICEF (2011), a adolescência pode representar uma grande oportunidade de transformação, quando se consideram as relações, as atitudes, a cultura, a educação, a vida e as dinâmicas sociais. Do ponto de vista jurídico, a adolescência é um período curto, durando apenas seis anos, dos 12 aos 18 anos incompletos. Toda essa complexidade permite valorizar a adolescência como uma fase singular e com um potencial imprescindível de transformação para a sociedade, na medida em que se favorece a vivência da adolescência em plenitude e possibilitar a chegada da fase adulta com maior maturidade.

No presente trabalho, parte-se do pressuposto de que a adolescência precisa ser entendida em toda a sua complexidade e deve ser atribuída ao adolescente a oportunidade para que ele mesmo construa sua autonomia e identidade, em múltiplas perspectivas e descobertas acerca de si mesmo. Desta forma, pensar sobre a sexualidade na adolescência exige um olhar em diversos sentidos, considerando principalmente as práticas sociais e os estilos de vida. Nesse sentido, o início da vida sexual circunscreve o adolescente no campo das vulnerabilidades em relação às doenças sexualmente transmissíveis, entre elas o HPV (BESERRA et al., 2008).

Chama a atenção o aumento da prevalência do câncer de cabeça e pescoço em adultos jovens, com presença de lesões associadas ao Papilomavírus Humano (Human papillomavirus - HPV). Recentemente, o HPV é considerado o maior fator de risco para este tipo de câncer e sua prevalência está em torno de 62\% (CARLANDER et al., 2017; SARKAR et al., 2017). A transmissão do HPV provém de práticas sexuais sem a devida proteção, especialmente exercidas durante o início da vida sexual. Tal constatação assinala para a necessidade de se promover ações preventivas durante a adolescência, período no qual a maioria das pessoas tem o início de sua vida sexual.

De forma geral, as campanhas de prevenção às doenças sexualmente transmissíveis são dirigidas e elaboradas sem se considerar o adolescente como ator do seu próprio processo. Neste trabalho, parte-se de um outro pressuposto, na medida em que se considera que qualquer iniciativa de educação ou prevenção deve ser implementada de forma participativa. Villela (1996) define a metodologia participativa como oficinas baseadas em técnicas lúdicas, vivências e dinâmicas em grupos. Segundo o autor, tal metodologia possibilita trabalhar, de forma simultânea, diversos aspectos relativos à sexualidade, articulando cognição, afeto, ideias, valores práticas e comportamentos.

Dispositivos móveis de nova geração, os Smartphones, representam hoje uma ferramenta de uso diário para uma boa parte da sociedade. Seu uso se espalhou por todo o mundo, embora existam diferenças significativas de seu uso entre as regiões, como se pode ver entre Europa Central e Oriente (151\%), Europa Ocidental (129\%) e América do Sul (124\%), regiões com a maior penetração destes dispositivos (LÓPEZ et al.; 2017).

Cabe destacar que os smartphones, além de atuar como facilitadores da comunicação, tanto intra como interpessoal, fornecem uma ampla gama de serviços relacionados com o 
entretenimento (jogos, música, televisão, etc.), informações (buscadores, blog especializado, etc.) e comunicação, com o uso das redes sociais (Twitter, Facebook, Instagram, Tinder, etc.). Essa versatilidade e funcionalidade, baseada em grande parte na sua natureza sem fio, converte o Smartphone em uma das tecnologias com o maior impacto na vida cotidiana e nas relações sociais, ampliando o próprio significado das mesmas (LÓPEZ et al.; 2017).

É inegável a atratividade que dispositivos móveis exercem sobre os adolescentes. Segundo a pesquisa TIC Kids Online Brasil (CETIC, 2016), o principal meio utilizado por eles para acessar a internet são os smartphones. Isso representa um aumento de $6 \%$ em um ano, a partir de 2015 , e de 38\% em três anos. A mesma pesquisa afirma que, em 2016, no Brasil, 22 milhões de crianças e adolescentes acessaram a internet por meio de celulares, sendo esse o único meio de acesso de $37 \%$ dos mesmos.

Desta forma, considera-se que o aplicativo para dispositivos móveis, em especial smartphones, é uma estratégia importante para ser utilizada na prevenção do HPV e, consequentemente, do câncer de cabeça e pescoço, tendo em vista o atual comportamento em relação à vacinação.

O movimento antivacinação foi incluído pela Organização Mundial de Saúde (OMS) em seu relatório sobre os dez maiores riscos à saúde global em 2019 (World Health Organization, 2019). De acordo com a OMS, os movimentos antivacina ameaçam reverter o progresso alcançado no combate a doenças evitáveis por vacinação, como o sarampo e a poliomielite. Nesta perspectiva o APP pode incentivar e promover a busca da vacina de HPV, pelos adolescentes. E ainda, por outra perspectiva, o APP pode instigar, no adolescente, a importância do cuidado com a saúde e com o corpo, e por consequência instigar, quem sabe, um cuidado permanente. Sendo assim, o objetivo deste trabalho foi desenvolver um aplicativo para dispositivos móveis (APP), voltado especialmente para adolescentes, como uma estratégia de prevenção ao HPV e ao câncer de cabeça e pescoço, a partir de uma metodologia participativa, e realizar a validação de conteúdo, aparência e aplicabilidade do APP.

\section{Materiais e métodos}

O presente trabalho foi desenvolvido com alunos do ensino médio do Instituto Federal de Educação, Ciência e Tecnologia do Paraná (IFPR). Situado na cidade de Irati, o câmpus possui $85 \%$ de seus 224 estudantes com faixa etária entre 12 e 19 anos, sendo $96 \%$ solteiros. Quanto à identidade de gênero, 50\% identificam-se como homens, $49 \%$ como mulheres e $1 \%$ como outros. Os estudantes dividem-se entre os cursos técnicos em Informática e Agroecologia integrados ao ensino médio e o curso superior em Tecnologia em Análise e Desenvolvimento de Sistemas.

Participaram 90 alunos de ensino médio, com idade entre 16 e 18 anos, de ambos os gêneros. Foram respeitados todos os preceitos éticos para a realização da pesquisa. Para um melhor entendimento do percurso metodológico realizado, as fases da pesquisa serão apresentadas em 4 etapas, sendo elas: 


\subsection{Etapa 1 - Concepção e elaboração do conteúdo do APP}

Esta etapa foi realizada a partir de metodologia participativa, em oficinas realizadas com os alunos. Para a concepção e elaboração do conteúdo do APP, foram realizadas oficinas participativas. Segundo Villela (1996), a oficina é sua proposta de aprendizagem compartilhada, em uma atividade em grupo, presencial, cujo objetivo é construir coletivamente o conhecimento. Os coordenadores apenas facilitam o debate, partindo sempre de dúvidas, opiniões e valores dos próprios participantes. Acredita-se que, a partir das discussões, os adolescentes podem ampliar seus recursos de autoproteção.

\subsection{Etapa 2 - Validação de conteúdo}

Segundo Alexandre e Coluci (2011), a validade de conteúdo é um processo de julgamento que envolve a análise de especialistas. No presente estudo, o conteúdo foi validado por três expertises, que possuem experiência na pesquisa e assistência em HPV e câncer de cabeça e pescoço, com nível de concordância maior do que $85 \%$.

\subsection{Etapa 3 - Desenvolvimento do APP}

Existem diversas metodologias de desenvolvimento de software. A vasta maioria especifica os passos que devem ser seguidos desde o processo de definição de requisitos (i.e., todas as funcionalidades que o software deve ser capaz de executar) até a validação da ferramenta (i.e., processo de verificação se o que foi desenvolvido é o que se desejava) e implantação (i.e., a forma de disponibilização do software para os usuários). Para o desenvolvimento do aplicativo a que esse trabalho se refere, empregou-se a Prototipagem Evolutiva. Tal metodologia é indicada quando o solicitante do programa possui pouca ou nenhuma experiência no desenvolvimento de aplicações (SBROCCO, 2012).

A primeira fase é a de "Comunicação". Nela, são levantados os requisitos do software através de uma ou mais reuniões com os stakeholders, ou seja, as pessoas envolvidas no desenvolvimento do projeto em todas as suas etapas, tais como clientes (quem está solicitando o aplicativo), engenheiros de software, programadores, administradores de banco de dados e designers.

A etapa do "Projeto Rápido" atua em conjunto com a de "Modelagem do Projeto Rápido" para definir quais aspectos, de preferência visíveis para o solicitante, serão prototipados. Na fase de "Construção do Protótipo", as partes selecionadas são implementadas. Como se optou pela variante evolutiva da Prototipagem, desenvolveu-se nesse momento um protótipo funcional, que já seja capaz (de imediato) de desempenhar as atividades escolhidas. 
Por fim, na etapa de "Emprego, Entrega e Realimentação", o protótipo é testado pelos solicitantes que, em reunião com a equipe de desenvolvimento, fazem suas considerações. Nessa reunião podem (e devem) surgir questões como a identificação de novos requisitos, correção na descrição de requisitos mal compreendidos ou mal expressados e discussão sobre elementos de interface. O resultado dessa reunião consiste na próxima fase de "Comunicação", tendo em vista que elas se repetem até que os solicitantes cheguem à conclusão de que o software atente a tudo aquilo que foi solicitado.

\subsection{Etapa 4 - Validação de aparência e aplicabilidade}

A análise de aparência e aplicabilidade tem o objetivo de verificar se o conteúdo é compreensivo para os membros da população ao qual se destina, se é claro, de fácil leitura e entendimento. Portanto, juízes na área temática do material educativo devem ser capazes de avaliá-lo, conferindo-lhe validade. A validade de aparência pode ser realizada por expertises e pela população alvo (ECHER, 2005). Na presente pesquisa, a validade de aparência foi realizada pelos próprios participantes da pesquisa, ou seja, pelos 90 estudantes do ensino médio.

\section{Resultados e Discussão}

\subsection{Etapa 1 - Concepção e elaboração do conteúdo do APP}

No presente estudo, as oficinas participativas foram realizadas em uma dinâmica entre os adolescentes. Cada oficina contou com a presença de aproximadamente 30 participantes, sendo então realizadas 3 oficinas com turmas diferentes. Os participantes foram dispostos em círculos e foram convidados a se apresentarem. A partir disso, foram realizadas as seguintes questões norteadoras: Você sabe o que é HPV? O que causa o HPV? O que o HPV tem a ver com o câncer?

As falas dos participantes foram anotadas na lousa, divididas em temáticas. Após o término dos depoimentos dos participantes, realizou-se uma demonstração de como ocorre a transmissão do HPV da região genital para a região oral e sua relação com o câncer de cabeça e pescoço. Além dos depoimentos, foi solicitado aos participantes que respondessem a questionários aplicados antes e após a realização das oficinas. Todas as informações adquiridas, tanto dos depoimentos quanto das respostas escritas, foram utilizadas para constituição do conteúdo do APP.

A demonstração da transmissão do HPV foi realizada por uma dinâmica participativa, na qual foi apresentada uma situação hipotética de uma "balada". Um grupo de 8 a 10 participantes assumiram os papéis de "baladeiros", sendo que um deles dispunha de uma folha de papel sulfite A4, que continha a representação do vírus HPV, com uma fita adesiva no centro. Este participante deveria "colar" o vírus HPV em um outro "baladeiro", de acordo com o contato sexual que pudesse ocorrer. Nas três dinâmicas, o local do corpo onde ocorreu a transmissão variou: na região da 
boca, na região das genitálias, na região anal e na região das mamas. Esta dinâmica foi acompanhada de muito riso e descontração por todos os participantes. Foi possível observar que a "transmissão do vírus" ocorreu entre e intergêneros, demostrando concretamente, toda pluralidade que os modos e relações permitem. Tal dinâmica serviu para demonstrar como ocorre a transmissão do HPV para a região oral e sua relação com o câncer de cabeça e pescoço, e como ela acontece de forma rápida e progressiva, potencializando o risco de contaminação.

Após a dinâmica participativa foi feita uma apresentação pelos coordenadores, esclarecendo o que é HPV, quantas linhagens de HPV existem, quais são oncogênicos e como ocorre a transmissão. A apresentação foi ilustrada com imagens da manifestação da doença causada pelo HPV, isto é, lesões nas genitálias e do câncer na região de pescoço, em específico na garganta. Foram enfatizadas, ao final da apresentação, as formas de prevenção, que são comuns para as doenças sexualmente transmissíveis e frisou-se que há disponível na rede pública de saúde, bem como na rede privada, uma vacina para as principais linhagens oncogênicas do HPV. Na rede pública, a vacina está disponível tanto para meninos, de 12 a 13 anos, como para meninas de 9 a 14 anos (BRASIL, 2017a).

Atualmente, a rotina de uso desta vacina no público-alvo é de 11 a 13 anos para meninos, faixa etária que será ampliada, gradativamente, até 2020, quando serão incluídos os meninos de 9 anos até 13 anos. Para as meninas, a faixa etária é mais ampla, sendo dos 9 aos 14 anos, com duas doses, sendo aplicada com intervalo de seis meses entre elas. A vacina do HPV é quadrivalente, contra as linhagens mais prevalentes e oncogênicas do HPV, as estirpes 6, 11, 16, 18. Ela é segura, eficaz e é a principal forma de prevenção contra o aparecimento do câncer do colo de útero, $4^{a}$ maior causa de morte entre as mulheres no Brasil. Nos homens protege contra os cânceres de pênis, orofaringe e ânus. Além disso, previne mais de 98\% das verrugas genitais, doença estigmatizante e de difícil tratamento (BRASIL, 2017b).

Contextualizando, as temáticas adquiridas pelas oficinas foram desenvolvidas, cientificamente, a partir de conhecimentos estabelecidos na literatura tomando como base livros e artigos indexados e qualificados. Criou-se o nome eduCO\&HPV, sendo edu referente à educação e CO a abreviação de câncer oral. Vale a pena ressaltar novamente que o conteúdo foi concebido pelos adolescentes e elaborado a partir desta demanda.

Este trabalho se alicerça nas ações de promoção de saúde dirigidas para adolescentes. Pensando nisso, optou-se por desenvolver um APP para smartphone, uma vez que essa tem sido uma das principais fontes de informações atualmente. Abrir espaços de produção de diálogo, reflexão e problematização junto à comunidade possibilita a construção de uma relação de corresponsabilidade, favorecendo formas mais humanas e efetivas do processo de prevenção em saúde (CAMARGO-BORGES; JAPUR, 2008).

A crescente inserção das tecnologias de informação e comunicação (TICs) no contexto da sala de aula, no atendimento educacional, a popularização dos dispositivos móveis e o crescente uso de interfaces sensíveis ao toque (touch screen) estão fazendo com que tais tecnologias estejam cada vez mais inseridas no contexto social das pessoas, seja pelo uso das novas gerações de telefones celulares ou pelo uso de dispositivos como tablets e lousas digitais interativas em atividades de ensino/aprendizagem nas escolas. Esses recursos propiciaram o surgimento de 
novas propostas metodológicas (jogos educativos, quizzes, etc), o que vem favorecendo aos poucos o desenvolvimento de sistemas com enfoque educativo, os quais podem facilitar 0 processo de ensino-aprendizagem de um grande número de pessoas, principalmente através de dispositivos mais populares como os telefones celulares. Esse novo processo educacional mediado por dispositivos móveis denomina-se M-Learning (LAOURIS; ETEOKLOUS, 2005; FAÇANHA et al., 2012; CROMPTON, 2013).

Outro fato que chama atenção é a metodologia utilizada neste estudo. O conteúdo desenvolvido foi obtido a partir das demandas dos participantes. As oficinas foram uma estratégia participativa e que levam em consideração os conhecimentos dos adolescentes. As novas tecnologias da informação e comunicação têm tido uma importância crescente na formatação das relações sociais, e são cada vez mais utilizadas como campo de pesquisa e intervenção em diversas áreas do conhecimento, em especial no campo da saúde coletiva (IRIART; SILVA, 2015).

$\mathrm{Na}$ oficina participativa o coordenador é um facilitador, orientador e mediador do processo de aprendizagem, estimulando a vivência de situações semelhantes àquelas que os estudantes vão encontrar na vida real. Os estudantes são responsáveis pelo seu processo de aprendizado, assumindo uma postura ativa e investigativa, construindo seu conhecimento em equipes de estudo e pesquisa (RODRIGUES et al, 2014).

Muitas vezes, as campanhas informativas e de prevenção partem do conhecimento daqueles que a elaboram e esta ação nem sempre contempla as necessidades do público-alvo. A presente pesquisa parte da premissa de que o tipo de abordagem na criação e execução de uma campanha é crucial para sua efetividade. Desta forma, buscou-se ser contundente em conhecer as necessidades reais dos adolescentes que utilizarão o aplicativo.

Em relação às temáticas sobre o HPV e o câncer de cabeça e pescoço, emergiram três temáticas principais: "O HPV e suas repercussões para a vida do adolescente"; "O câncer de cabeça e pescoço e sua relação com o HPV"; "HPV e câncer de cabeça e pescoço: como prevenir?". As três temáticas reveladas nas falas dos adolescentes foram o ponto de partida para a elaboração do conteúdo do APP.

Interessante apresentar alguns depoimentos dos adolescentes envolvendo as questões de gênero, como se vê a seguir:

"Eu achava que o HPV só dava em mulheres"

E. 15

"A vacina é para homem também? Eu achava que só menina podia tomar" E. 67

Os depoimentos acima referem-se à associação entre o HPV e o câncer em mulheres, com especial destaque ao câncer de colo de útero. O câncer de colo de útero é um sério problema de saúde pública no Brasil, atingindo principalmente as mulheres mais pobres, com maior dificuldade de acesso aos serviços de saúde. É o segundo tipo de câncer mais comum na população feminina brasileira e corresponde à quarta causa de morte de mulheres, por câncer, no país (INCA, 2017). 
Há décadas são realizadas campanhas dirigidas às mulheres, com o intuito de se prevenir este tipo de câncer. Historicamente, a partir da década de 1930, os médicos começaram a valorizar as ações educativas informativas sobre câncer, com ênfase no seu diagnóstico precoce, tendo em vista maiores chances de cura na fase inicial da doença. Nesta década foram criados os primeiros materiais educativos, com incentivo de Mário Kroeff, criador do Centro de Cancerologia (1937), hoje Instituto Nacional de Câncer José Alencar Gomes da Silva (INCA). Porém, foi em meados da década de 1960 que os médicos brasileiros começaram a realizar campanhas de rastreamento do câncer do colo de útero, utilizando o teste Papanicolau. Somente na década de 1970, especificamente em 1977, foi sugerido por zur Hausen a existência de relação entre o HPV e o câncer do colo do útero, sendo esta comprovada ao longo da década de 1980, com o isolamento do vírus em células de tumores cervicais (CORRÊA; RUSSOMANO, 2012; zur HAUSEN, 2002).

Assim, fica evidente que ocorre uma certa cristalização da relação do HPV e o câncer de colo de útero $e$, consequentemente, ao gênero feminino, visto que há toda uma trajetória histórica e de grande relevância para a saúde humana. Cabe a ressalva de que o HPV não tem restrições de sexo, isto é, ele infecta tanto homens, quanto mulheres, e causa os mesmos tipos de cânceres, com distinção apenas anatômica, quando se restringe aos aparelhos reprodutores. Na mulher, o HPV causa câncer de colo de útero e genitália e no homem somente de genitália.

Atualmente, no âmbito da saúde, são concebidas várias campanhas informativas, educativas e preventivas (LIMA et al., 2017), mobilizadas inicialmente por sociedades médicas, pacientes e ONGs, com ampla divulgação nas mídias e grande repercussão, até mesmo mundial. Como exemplo, temos o Outubro Rosa, com foco na prevenção do câncer de mama, o Novembro Azul de atenção ao câncer de próstata; o Dezembro Laranja para o câncer de pele; o Janeiro Roxo/Lilás para o combate ao câncer de colo de útero; e o Março Azul-marinho para o câncer de intestino.

Dentro deste contexto de demandas surgiu em 2014, no Congresso Mundial de Câncer de Cabeça e Pescoço, uma campanha de combate a este tipo de câncer que ficou versada como Julho Verde. O Julho Verde recebeu uma adesão um tanto tímida no Brasil, apesar da alta prevalência desta enfermidade. A campanha serviu de motivação para a criação do APP para smartphones, como uma tentativa de ampliar a divulgação de informações sobre o câncer de cabeça e pescoço.

É de se destacar que, ao promover uma oficina participativa, tendo como tema uma doença sexualmente transmissível, ocorrem fundamentações de cunho de educação sexual. Portanto, a concepção e a construção do APP proporcionaram uma abrangência muito além daquela inicialmente pré-concebida, afinal, não há como separar a parte do todo. Os assuntos são intrínsecos e entrelaçados e têm, inexoravelmente, uma ordem interdisciplinar, pois se trata não somente de uma doença, mas envolve comportamentos de sujeitos sociais, repletos de experiências e significados.

Muitos participantes fizeram comentários, em seus relatos escritos pós-oficinas, referentes aos seus comportamentos sexuais e de novas perspectivas dirigidas para o cuidado com o próprio corpo e de exposição dele de forma mais segura, a partir do conhecimento construído durante 
as oficinas. Tais depoimentos apontam que estas iniciativas participativas são ações efetivas, que promovem a produção de um conhecimento não estéril, e sim emancipatório e agregador.

Vale a pena destacar também que os participantes valorizaram a criação de um APP como uma estratégia de orientação e abordagem, já que o uso de smartphone está presente no cotidiano da maioria dos adolescentes. Os participantes solicitaram que os conteúdos fossem apresentados com uso de imagens sobre o HPV e o câncer de cabeça e pescoço. Acredita-se que o APP é uma opção assertiva e que pode atingir um número grande de pessoas.

Ressalta-se que o APP pode agir como incentivador ao usuário na busca de prevenção e ou cura, na medida em que ele problematiza a questão da doença. O APP poderá servir de adjuvante nas campanhas de vacinação contra o HPV e incentivá-las. O governo federal do Brasil lançou, em Abril de 2016, uma campanha para incentivar as meninas na procura pela vacina. Entretanto, em 2017, observou-se uma nova campanha motivada pela "sobra" de vacinas na rede (BRASIL, 2017b). Diante de um estoque com mais de 64 mil doses de vacina contra HPV prestes a perder a validade, o Ministério da Saúde decidiu afrouxar as regras e permitir que homens e mulheres de até 26 anos recebam também a vacina (BRASIL, 2017b).

Apesar de todos esses esforços, as coberturas vacinais continuam abaixo da meta preconizada de $80 \%$. Isso se dá porque a vacinação na adolescência tem uma série de dificuldades, como a resistência desse grupo etário de buscar uma unidade de saúde, especialmente para vacinar-se, além do baixo conhecimento sobre a importância da vacinação (BRASIL, 2017b).

Durante as oficinas, muitas meninas relataram, por motivos diversos, não terem se vacinado. Portanto, o APP poderia auxiliar esse aspecto, informando as adolescentes sobre a vacina e diminuindo a desinformação e a resistência delas, ajudando nas campanhas e fazendo ele próprio uma "campanha" de busca à vacina.

Nota-se novamente o quão efetivo e profícuo é trabalhar uma oficina e prospectar o conhecimento. A oficina demonstrou ser uma estratégia participativa e um momento de discussão dos conflitos, dúvidas, receios e medos, além da carência de informações dos estudantes. O presente trabalho parte então de uma outra lógica, considerando as demandas do público-alvo, ou seja, dos próprios adolescentes.

Acredita-se na importância das campanhas e também da vacinação. Mas, para além dessas ações, destaca-se as oficinas e o APP como estratégias importantes para lidar com os adolescentes, pois existem outros fatores coadjuvantes na efetivação da prevenção do HPV e, neste contexto, as oficinas e o APP têm grande efetividade de aproximação com os adolescentes.

Neste cenário contemporâneo, observa-se ainda que, com as novas possibilidades interativas e conexões diversas, surgem também novos "sujeitos" da saúde coletiva (profissionais, pesquisadores, comunidades etc.). E isso aponta para a necessidade de se conhecer melhor esses "actantes" (pessoas e tecnologias), como também de discussão e revisão contínua dos limites e potencialidades de nossas políticas públicas (IRIART; SILVA, 2015) e de planejamento educacional.

\subsection{Etapa 2 - Validação de conteúdo}


Participaram da validação de conteúdo três juízes expertises na área. Os três juízes possuem mais de vinte anos de atuação na temática HPV e câncer de cabeça e pescoço e titulação de Doutor. Todo o conteúdo do aplicativo foi analisado e cada tela contou com a concordância maior ou igual a 85\%. Assim, os conteúdos do APP foram sobre: o que é o HPV, o que é o câncer de cabeça e pescoço, a relação entre o HPV e o câncer de cabeça e pescoço, formas de contágio e prevenção, sendo que todas as informações foram ilustradas com fotos e imagens de domínio público, para chamar a atenção da população alvo. Constam ainda no APP locais onde o usuário pode procurar orientações e atendimentos, caso seja necessário.

\section{3 - Etapa 3 - Desenvolvimento do APP}

O aplicativo desenvolvido, denominado educo\&HPV, possui as seguintes funcionalidades: (1) apresentação de cartões em formato de slider contendo informações sobre o HPV bem como sua relação com o câncer de cabeça e pescoço; (2) exibição de questionário para pesquisa georreferenciada com questões sobre gênero, idade, hábitos sexuais e consumo de álcool e tabaco (cigarro e narguilé); (3) disponibilização de links para páginas na Web nas quais podem ser encontradas mais informações sobre o assunto e também pesquisas científicas sobre a temática (PRESSMANN, 2011).

A Figura 1 mostra a tela de apresentação do APP, a 2 a tela inicial, que contém um menu no qual o usuário pode selecionar uma opção e navegar entre informações sobre o HPV (Figura 3a), informações sobre o Câncer de Cabeça e de Pescoço (Figura 3b), sobre a relação entre o HPV e o Câncer de Cabeça e de Pescoço, bem como sobre o modo como isso pode atingi-lo. O usuário pode, ainda, participar de uma pesquisa (Figura 4) ou acessar informações sobre o projeto.

Figura 1 - Tela de apresentação do aplicativo.

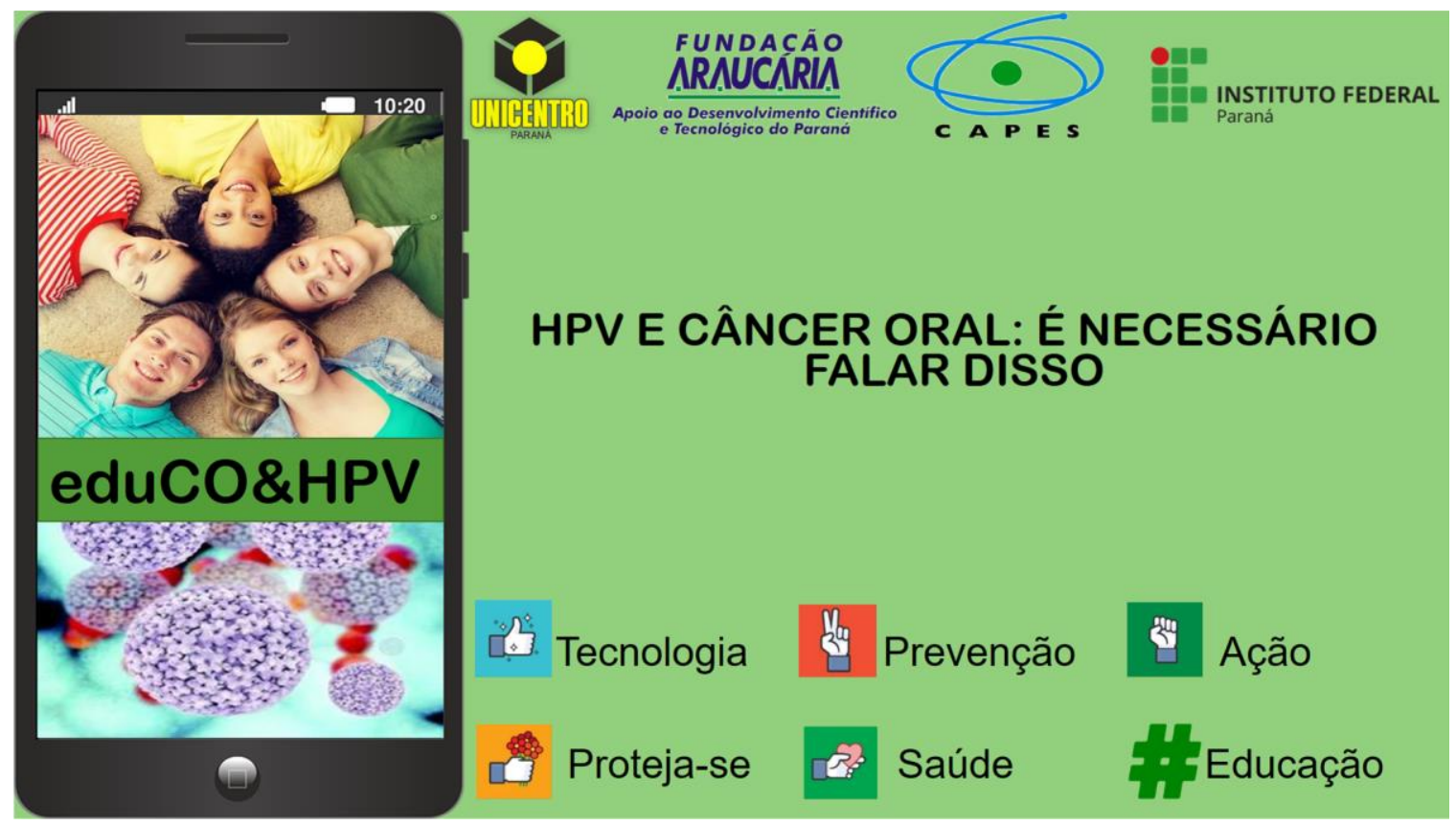



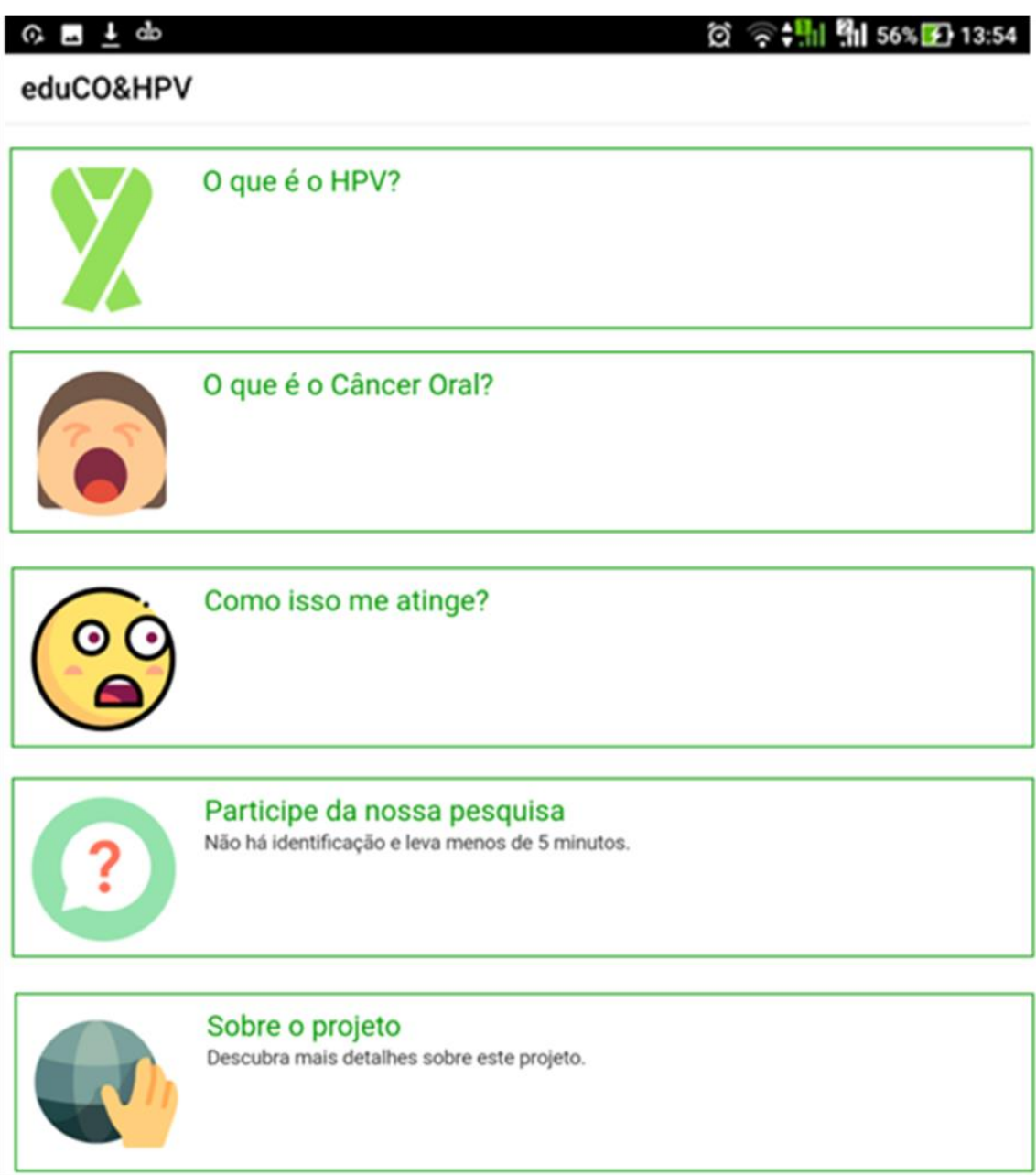

Fonte: Autor.

Figura 3 - Telas mostrando cartões informativos apresentados em estrutura de slides. Em (a) temos a exibição de imagens e em (b) de texto em formato resumido.

(a)

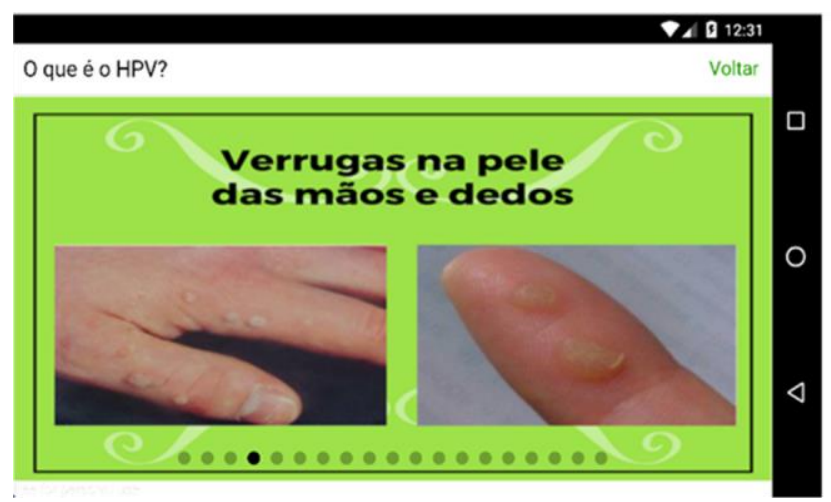

(b)

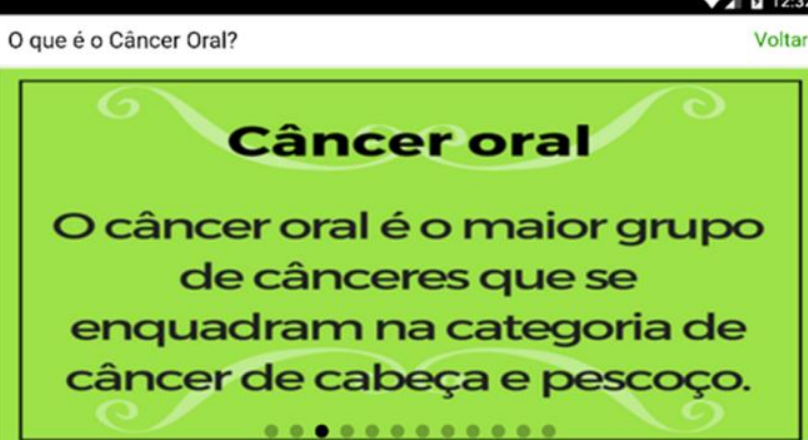


Figura 4 - Tela mostrando um fragmento do questionário.

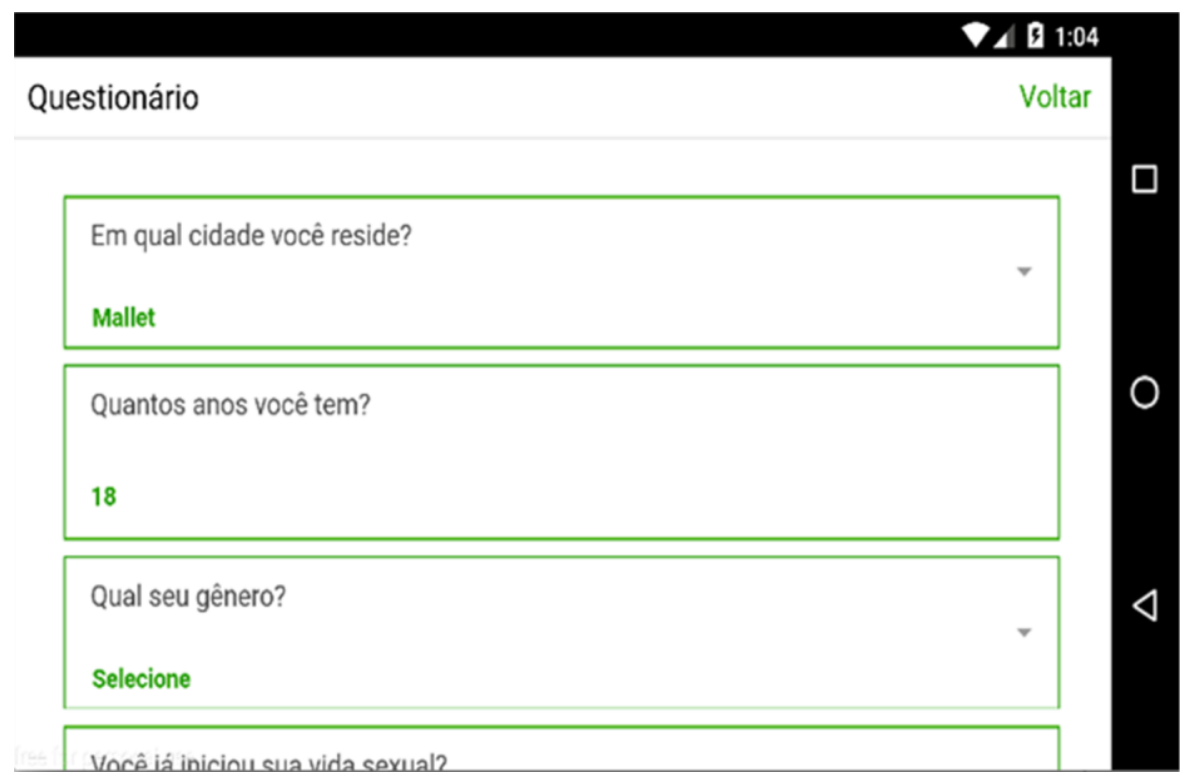

Fonte: Autor.

Optou-se por uma estrutura com poucos elementos e cores para dar ênfase ao conteúdo da aplicação. Para a formatação dos cartões foi escolhida uma apresentação com padrão de cores, fontes e limitação da quantidade de texto com restrição às ideias centrais, visando uma adequação ao público-alvo os adolescentes, que estão habituados a informações curtas e diretas. Os detalhes considerados muito técnicos sobre o assunto foram deixados para consulta em links disponibilizados na opção "Sobre o projeto" da tela inicial. Estas decisões de projeto foram validadas conforme descrito nos resultados da Etapa 4, sobre a validação de aparência do APP.

$\mathrm{Na}$ interface do questionário, quando o usuário envia suas respostas o aplicativo se conecta a um Web Service responsável por receber os dados e armazená-los em um banco de dados para pesquisas e relatórios futuros.

\subsection{Etapa 4 - Validação de aparência e aplicabilidade}

Na presente pesquisa, a validade de aparência e aplicabilidade foi realizada pelos próprios participantes da pesquisa, em duas etapas, e contou com concordância maior ou igual a $85 \%$. 
Na primeira etapa, os adolescentes utilizaram a primeira versão do APP, e solicitaram mudanças no conteúdo, com especial destaque à utilização de termos considerados "muito científicos", excesso de conteúdos escritos nas telas, além de questões referentes ao layout como fonte, cor da fonte, cor do fundo, entre outras.

Todas as solicitações dos adolescentes foram atendidas. Numa segunda etapa, os adolescentes concordaram com as mudanças realizadas e apontaram a importância da criação do APP, considerando, inclusive, que é mais fácil para eles tirarem dúvidas e seguirem orientações via aplicativo uma vez que eles acreditam que se fossem questionar uma pessoa certamente seriam "julgados". Esse tipo de julgamento não ocorre com o aplicativo e os adolescentes disseram se sentir mais "livres".

\section{Considerações Finais}

O aplicativo eduCO\&HPV foi desenvolvido a partir de uma metodologia participativa. Os conteúdos do aplicativo foram concebidos a partir de uma oficina realizada com adolescentes e foram validados por expertises na área, com concordância maior ou igual à 85\%. A validação de aparência e aplicabilidade foi realizada pelos próprios adolescentes e também contou com concordância maior ou igual à $85 \%$.

A oficina demonstrou ser uma estratégia importante para o desenvolvimento do aplicativo, uma vez que as informações obtidas na sua execução foram extremamente relevantes para conceber o conteúdo do APP. Durante a validação de aparência e aplicabilidade, o APP foi muito bem avaliado pelos adolescentes como estratégia de divulgação de informações da relação entre o HPV e o câncer de cabeça e pescoço.

O APP eduCO\&HPV já está disponível para download gratuito na loja Google Play. Tais ações são importantes para prevenir futuros casos de pacientes com câncer de cabeça e pescoço, para promover orientação na educação sexual e criar novas possibilidades de intervenção, não só em nível de saúde, mas de educação, na sua mais estrita concepção.

\section{Referências}

ALEXANDRE, N.M.C.; COLUCI, M.Z.O. Validade de conteúdo nos processos de construção e adaptação de instrumentos de medidas. Ciênc. saúde coletiva, v.16, n.7, p.3061-3068, 2011.

BESERRA, E.P.; PINHEIRO, P.N.C.; ALVES, M.D.S.; BARROSO, M.G.T. Adolescência e vulnerabilidade às doenças sexualmente transmissíveis: uma pesquisa documental. J Bras Doenças Sex Transm. V.20, n.1, p.32-5, 2008.

BRASIL. Ministério da Saúde. Saúde amplia vacina de HPV para meninos de 11 até 15 anos incompletos. Disponível em: < http://portalarquivos.saude.gov.br/images/pdf/2017/junho/20/Anuncio-ampliacao-HPVpara-meninos.pdf>. Acesso em: 21/11/2017a.

BRASIL. MINISTÉRIO DA SAÚDE. Homens e mulheres entre 15 e 26 anos podem se vacinar contra HPV até março. Disponível em: <http://portalms.saude.gov.br/noticias/agencia-saude/29526-homens-e-mulheresentre-15-e-26-anos-podem-se-vacinar-contra-hpv-ate-marco>. Acesso em: 21/11/2017b. 
CAMARGO-BORGES, C; JAPUR, M. Sobre a (não) adesão ao tratamento: ampliando sentidos do autocuidado. Texto e Contexto Enferm., v.17, n.1, p.64-71, 2008.

CARLANDER, A. F.; LARSEN, C. G.; JENSEN, D. H.; GARN/ES, E; KISS, K; ANDERSEN, L; OLSEN, C. H.; FRANZMANN, M; HØGDALL, E; KJÆER, S. K.; NORRILD, B; SPECHT, L; ANDERSEN, E; VAN OVEREEM HANSEN, T; NIELSEN, F. C.; VON BUCHWALD, C. Continuing rise in oropharyngeal cancer in a high HPV prevalence area: A Danish population-based study from 2011 to 2014. Eur J Cancer., v.70, p.75-82, 2017.

CENTRO REGIONAL PARA O DESENVOLVIMENTO DA SOCIEDADE DA INFORMAÇÃO-CETIC. Pesquisa sobre o uso da internet por crianças e adolescentes no Brasil. São Paulo: Comitê Gestor da Internet no Brasil, 2016. Disponível em:

<http://cetic.br/media/docs/publicacoes/2/TIC_Kids_2015_LIVRO_ELETRONICO.pdf>.

CORRÊA, F.M; RUSSOMANO, F.B. Novas tecnologias de prevenção do câncer do colo do útero: quem de fato se beneficia? Revista Brasileira de Cancerologia, v.58, n.3, p. 525-527, 2012.

CROMPTON, H. A historical overview of M-Learning. In: BERGE, Z.; MUILENBURG, L. (Eds). Handbook of mobile learning. New York: Routledge, 2013. p. 3-14.

ECHER, I.C. The development of handbooks of health care guidelines. Rev Lat Am Enfermagem, v.13, n.5, p.754-757, 2005.

FAÇANHA, A.R.; LIMA, L.S.; ARAÚJO, M.C.C.; CARVALHO, W.V.; PEQUENO, M. C. Auxiliando o Processo de Ensino-Aprendizagem do Braille Através de Dispositivos Touch Screen. Informática na Educação: teoria \& prática, v.15, n.2, p.153-169, 2012.

INSTITUTO NACIONAL DO CÂNCER (INCA). Controle do Câncer do Colo de útero - Campanhas de Prevenção do Câncer do Colo do Útero no Brasil, 2017. Disponível em:

<http://www2.inca.gov.br/wps/wcm/connect/acoes_programas/site/home/nobrasil/programa_nacional_co ntrole_cancer_colo_utero/impressos-multimidia/campanhas-de-prevencao-do-cancer-do-colo-do-utero-nobrasil>. Acesso em: 23/10/2017.

IRIART, J.A.B.; SILVA, L.A.V. As tecnologias da informação e comunicação e novas formas de sociabilidade: possibilidades e desafios para o campo da saúde coletiva. Cad. Saúde Pública, v.31, n.11, p. 2253-2254, 2015.

LAOURIS, Y.; ETEOKLEOUS, K. We need an educationally relevant definition of mobile learning. In: Proceedings of mLearn, 2005.

LIMA, A.C.M.A.C.C. et al. Construção e validação de cartilha para prevenção da transmissão vertical do HIV. Acta paul. enferm., v.30, n.2, p.181-189, 2017.

LÓPEZ, M.A.; GUTIÉRREZ, V.F.; GARCÍA-DOMINGO, M. "No sin mi Smartphone": Elaboración y validación de la Escala de Dependencia y Adicción al Smartphone (EDAS). Ter Psicol, v.35, n.1, p. 35-45, 2017.

MORAES, S.P.; VITALLE, M.S.S. Direitos sexuais e reprodutivos na adolescência. Rev. Assoc. Med. Bras., v. 58, n. 1 , p. $48-52,2012$.

PRESSMAN, R.S. Engenharia de software: uma abordagem profissional.Porto Alegre: Bookman, 2011.

RODRIGUES, A. H.; MARTINS, P. H. M. L.; CAMPOS, V. D.; GNATKOSKI, T. F. Oficinas de aprendizagem no contexto do ensino médio: relato de experiência. In: KAZAMA, R.; PEDRO, J.M.; MIGUEL, R.B.P.; VARVAKIS, G.; BENTANCOURT, S.M.P; SILVA, M.P.; PEREIRA, M.S.; LOZANO, L.; CARDENAS, Y.G. (Org.). Interdisciplinaridade: teoria e prática. $1^{a}$ ed. Florianópolis: Editora UFSC/EGC, 2014.

SARKAR, S; ALAM, N; CHAKRABORTY, J; BISWAS, J; MANDAL, S. S.; ROYCHOUDHURY, S; PANDA, C. K. Human papilloma virus (HPV) infection leads to the development of head and neck lesions but offers better prognosis in malignant Indian patients. Med Microbiol Immunol., v.206, n.3, p.267-276, 2017.

SBROCCO, J.H.T.C.; MACEDO, P. Metodologias Ágeis-Engenharia de Software sob medida. São Paulo: Editora Érica Ltda, 2012.

UNICEF. Situação Mundial da Infância. Adolescência: uma fase de oportunidades. Genebra: UNICEF, 2011. 
VILLELA, W. Oficinas de sexo mais seguro para mulheres. Abordagens metodológicas e de avaliação. São Paulo: NEPAIDS-USP, 1996.

WORLD HEALTH ORGANIZATION. Ten threats to global health in 2019. Disponível em: <

https://www.who.int/emergencies/ten-threats-to-global-health-in-2019>. Acesso em: 23/09/2019.

zUr HAUSEN, H. Papillomaviruses and cancer: from basic studies to clinical application. Nat Rev Cancer., v.2, n.5, p.342-50, 2002.

Recebido em setembro de 2018

Aprovado para publicação em agosto de 2019.

\section{Alcir Humberto Rodrigues}

Programa de Pós Graduação Interdisciplinar em Desenvolvimento Comunitário (PPGDC) da Universidade Estadual do Centro-Oeste (UNICENTRO), Irati - PR, Brasil, alcirhumberto@gmail.com

\section{Elaine Fátima Brek}

Departamento de Fonoaudiologia da Universidade Estadual do Centro-Oeste (UNICENTRO), Irati - PR, Brasil, elaine-808@hotmail.com

\section{Flavia Valenga}

Departamento de Fonoaudiologia da Universidade Estadual do Centro-Oeste (UNICENTRO), Irati - PR, Brasil, flaviavalenga@hotmail.com

\section{Cleverson Sebastião dos Anjos}

Departamento de Informática do Instituto Federal de Educação, Ciência e Tecnologia do Paraná (IFPR), Irati - PR, Brasil, cleverson.anjos@ifpr.edu.br

\section{Valter Luís Estevam Junior}

Departamento de Informática do Instituto Federal de Educação, Ciência e Tecnologia do Paraná (IFPR), Irati - PR, Brasil, valter.junior@ifpr.edu.br

\section{David Livingstone Alves Figueiredo}

Departamento de Medicina e Programa de Pós Graduação Interdisciplinar em Desenvolvimento Comunitário (PPGDC) da Universidade Estadual do Centro-Oeste (UNICENTRO), Guarapuava - PR, Brasil, davidlafigueiredo@gmail.com

\section{Cristina Ide Fujinaga}

Departamento de Fonoaudiologia e Programa de Pós Graduação Interdisciplinar em Desenvolvimento Comunitário (PPGDC) da Universidade Estadual do Centro-Oeste (UNICENTRO), Irati - PR, Brasil, cifujinaga@gmail.com

\section{Agradecimentos}

O presente trabalho foi realizado com apoio da Coordenação de Aperfeiçoamento de Pessoal de Nível Superior - Brasil (CAPES) - Código de Financiamento 001. 